Continents manuscrits

Génétique des textes littéraires - Afrique, Caraïbe, diaspora

12 | 2019

Génétique et anthropologie

\title{
La double écriture
}

Joseph Tonda

Édition électronique
URL : https://journals.openedition.org/coma/4086

DOI : $10.4000 /$ coma.4086

ISSN : 2275-1742

\section{Éditeur}

Institut des textes \& manuscrits modernes (ITEM)

\section{Référence électronique}

Joseph Tonda, «La double écriture », Continents manuscrits [En ligne], 12 | 2019, mis en ligne le 10 septembre 2019, consulté le 14 janvier 2023. URL : http://journals.openedition.org/coma/4086 ; DOI : https://doi.org/10.4000/coma.4086

Ce document a été généré automatiquement le 14 janvier 2023.

\section{(c) (i) $(9)$}

Creative Commons - Attribution - Pas d'Utilisation Commerciale - Pas de Modification 4.0 International - CC BY-NC-ND 4.0

https://creativecommons.org/licenses/by-nc-nd/4.0/ 


\title{
La double écriture
}

\author{
Joseph Tonda
}

1 Les prophètes sont des créateurs de réalités que les sciences humaines se chargent de convertir en objets scientifiques, exprimant ainsi leur inextinguible dette à l'endroit de ces démiurges. Et c'est parce que cette dette est inextinguible, que le sociologue ou l'anthropologue, hantés par "le syndrome du prophète ${ }^{1}$ ", éprouvent au fond d'euxmêmes une douloureuse insatisfaction de leur travail de conversion de la réalité créée par le geste prophétique en objet de connaissance scientifique. Il y a en effet ce reste inépuisable du discours de l'Autre créateur de réalité que l'on ne saurait dompter, et que l'on s'imagine consciemment ou peut-être surtout inconsciemment pouvoir vaincre en se lançant soi-même dans l'aventure de la double création. Création littéraire, mais aussi création cinématographique sous la forme du film documentaire ethnographique que l'on écrit et produit ou que la production du scientifique inspire, redoublant ainsi la force de ses manuscrits.

2 Entreprise très risquée que celle du sociologue ou de l'anthropologue qui, pour l'occasion, se transforme en écrivain afin de créer LA réalité dont la charge épistémologique de la description, de l'analyse et de l'interprétation incombera à ses collègues ou à lui-même, en fonction du cadrage théorique adopté. Si l'on parle bien de construction de l'objet c'est bien parce que, selon Gaston Bachelard, la démarche scientifique consiste à rompre avec le «sens commun", cette réalité partagée par la pensée commune, dite aussi pensée ordinaire, que le romancier met en scène dans sa création.

3 Le scientifique doit, par la construction de son objet, faire éclater ce qui paraît évident pour faire voir (= révéler) ce qui est derrière cette évidence. Sa démarche est alors analogue à celle du psychanalyste qui doit mettre au jour la réalité latente du rêve qui se cache derrière la réalité manifeste. Cela n'est possible qu'en s'inscrivant dans un cadre théorique qui doit guider l'analyse des données empiriques qui, s'agissant du contenu d'un roman, est constituée par la mise en scène de sa pensée. Le résumé de ce précepte épistémologique est le suivant : le fait scientifique est conquis sur les préjugés (on pense alors que la création du romancier comprend ces « préjugés »); construit par 
la raison (la théorie ou le cadre analytique) et constaté par les faits (la réalité de la société ayant inspiré le romancier).

4 Cette construction a d'autant plus de chance d'obtenir la reconnaissance scientifique que l'auteur fait preuve d'imagination sociologique ou anthropologique. Mais que signifie en réalité faire preuve d'imagination si ce n'est se faire auteur au sens où l'on parle de l'auteur d'un rêve? Car selon Pierre Bourdieu, «Les "sujets sociaux" seraient beaucoup plus sujets s'ils savaient qu'ils sont très rarement sujets et qu'en fait "ça" parle. ${ }^{2}$ " Le sociologue ou l'anthropologue échapperaient-ils à ce "ça » qui pense et qui parle en eux? En ce qui concerne le prophète, le problème est résolu, car il dit luimême le nom de celui qui parle en lui. Sa parole ne lui appartient pas, et s'il accomplit des miracles, il le fait au nom de ceux-là qui parlent en lui, qui l'habitent, et qui guident sa pensée, sa parole, ses actes. Effectivement, « Ça parle en lui ». Or, il en est de même de l'écrivain, qui dans son expérience de l'écriture, se sent transporté parce qu'il est "inspiré»; et les personnages qu'il a créés prennent progressivement une sorte d'existence autonome qui résiste aux décisions de l'auteur. Cette expérience a donné lieu à des mythes têtus, dont celui de Frankenstein : la créature qui s'autonomise et qui se retourne contre son créateur. Mon propos consiste à montrer comment la création de la réalité coloniale et postcoloniale repose sur la puissance du «ça » qui «pense ", qui «parle» et qui «fait» agir aussi bien les prophètes que les chercheurs, les chefs d'État et les artistes, et ceci, sous le régime paradigmatique de la double écriture.

\section{La violence de l'imaginaire prophétique, expression de l'afrodystopie}

5 J'avais entre 5 et 6 ans quand je fus confronté à la violence prophétique. Avec le temps et avec ma formation de chercheur en sciences sociales, je puis affirmer que dans cette cité ouvrière rurale de Lébango, dans le Nord-Ouest congolais, je vécus au cours de cette douloureuse expérience la réalité de l'inséparabilité du réel et de l'imaginaire. Une expérience physique de ce que j'appelle la violence de l'imaginaire ${ }^{3}$. En effet, l'agent de cette violence alimentée par l'inséparabilité du réel et de l'imaginaire fut le prophète du culte Mademoiselle, qui vit le jour entre 1953 et 1954, et dont le fondateur fut Emane Boncœur, un Congolais originaire de la région de la Sangha, dans le NordCongo (Brazzaville) ${ }^{4}$. L'un des plus puissants disciples d'Emane Boncœur fut Pascal Zoaka Zoaka, originaire de la province de l'Ogooué-Ivindo, au Gabon, qui entreprit, sous le commandement des esprits dénommés Jean et Mademoiselle, une violente campagne de défétichage et de lutte contre la sorcellerie (Izanga ${ }^{5}$, en langue ikota, dont il fut locuteur) sur les anciens territoires du Moyen-Congo et du Gabon. Le défétichage n'épargnait même pas les reliquaires kota, surmontés du fameux masque qui fut précipité dans les profondeurs des rivières. Pour celui qui se nomma lui-même Monseigneur Pascal Zoaka Zoaka, la figure du masque kota fut un bwele, que l'on peut traduire par «médicament maléfique » qu'il fallait détruire pour libérer la société des forces de la nuit qu'elle représentait. En d'autres termes, les « objets d'art africains » qui font débat de nos jours étaient considérés par les prophètes comme des objets de malheur, autrement dit, des objets caractéristiques de l'Afrodystopie, la dystopie africaine.

6 Si l'on tient à cette logique prophétique, force est de constater que le combat des intellectuels africains pour le retour de ces objets en Afrique viserait à faire revenir sur 
le continent ce que les prophètes africains comme Malanda au Congo-Brazzaville et Pascal Zoaka Zoaka au Gabon considéraient comme des objets diaboliques sous la pensée des esprits du christianisme au nom desquels ils prêchaient. Ainsi, selon ces esprits qui s'exprimaient par leur voix, les Noirs étaient plongés dans le noir des ténèbres par ces «fétiches » de leur fabrication. Ce qui veut dire qu'en détruisant ces poisons psychiques, les Noirs sortiraient de la « fausse conscience » pour entrer dans la lumière dont le vecteur ne pouvait être, dans la logique des pensées prophétiques d'un Malanda ou d'un Pascal Zoaka Zoaka, que le christianisme. Une lumière représentée par la pensée nourrie par ce que nous appelons ici la double écriture.

7 En effet, par cette expression, nous entendons l'ensemble formé par les écritures au sens comptable, où l'on dit «tenir les écritures ", mais aussi, par les Saintes Écritures qui renvoient aux textes de l'Ancien et du Nouveau Testament. Il est significatif, dans cette perspective, que cette notion d'écritures, dans sa dimension comptable, proroge et accentue la représentation que les Gabonais ont eue des Blancs : Otangani, c'est-à-dire " ceux qui comptent ${ }^{6}$ ». Il s'agit de ces personnes " venues d'ailleurs», et qui, dans les villages, demandaient aux populations le nombre de leurs enfants ; sans se soucier de ce que cette notion d'enfants signifiait pour leurs protagonistes. S'agissait-il, pour un homme, des enfants nés de son union avec son épouse, ou des enfants de sa sœur, qui étaient aussi ses enfants, notamment dans les structures de filiation matrilinéaire ? De manière plus générale, la réponse à la question « Tu as combien d'enfants? » soulevait d'autres problèmes au sujet des rapports de parenté, par exemple, elle posait le problème de qui est une sœur pour un homme et qui ne l'est pas, dès lors que, pour un homme interrogé, au nombre de ses sœurs, il y a ce que l'on appelle en Europe les " cousines ». Dans une structure de filiation matrilinéaire, les enfants des « cousines » nées de la sœur de la mère d'égo sont ses enfants... Ces enfants-là n'étaient pas moins "biologiques" que les autres. Au-delà, les problèmes que posait la comptabilité des hommes et des femmes se répercutaient dans ceux soulevés par le nombre de poules ou d'autres biens. L'«homme qui compte", l'Otangani, le Ntangan ou le Ntang, selon les langues ethniques, c'était ainsi cet homme blanc, soucieux de faire le recensement des hommes, des femmes, des enfants, des biens, à des fins d'assujettissement dont l'un des instruments coercitifs était l'impôt de capitation. Cet « homme qui compte » qui avait la figure de l'administration, c'était aussi, le prêtre ou le pasteur, ceux-là qui comptaient leurs ouailles, pour le paiement de la dime. Un roman de Mongo Beti, Le Pauvre Christ de Bomba, décrit de manière fort saisissante cette logique comptable du missionnaire fondée sur les Écritures au sens religieux.

\section{La double écriture prophétique}

8 La double écriture est ainsi cet ensemble formé par les écritures comptables et les Écritures saintes, et qui met en exergue la matérialité de l'idéologie qui se traduit par la violence de l'imaginaire, dont Monseigneur Pascal fut un terrible agent de fonction pour les populations qui furent exposées à son action. Jean et Mademoiselle, esprits qui pensaient en Pascal Zoaka Zoaka et commandaient ses actes de violence, se situaient dans l'imaginaire prophétique fondé sur les Écritures saintes, comme l'atteste le fait que la carrière de ce prophète commença par la revendication d'un livre: il lui fut destiné par l'esprit Jean qui le confia à Marion, un chef blanc du chantier aurifère où fut employé Pascal. L'imaginaire missionnaire, qui se trouve ainsi recyclé par le 
prophétisme de Monseigneur Pascal, reposait quant à lui sur les deux écritures, et de nos jours, l'imaginaire des prophètes-pasteurs reproduit à la fois l'imaginaire des prophètes et celui des missionnaires : la violence psychique matérialisée par l'argent de la dime, la violence indifféremment psychique et physique des délivrances ou des exorcismes, étant la matérialisation de la consubstantialité de l'imaginaire et du physique dans la violence de l'imaginaire. Portés par cette violence de l'imaginaire de la double écriture, prêtres catholiques, pasteurs protestants et leurs homologues prophètes africains dont les combats sont fermement repris en main aujourd'hui par les pasteurs-prophètes pentecôtistes, qui prêtent tous foi aux Écritures, et donc à la double écriture, sont inscrits dans la dynamique de la production du «nouveau sujet africain », débarrassé des objets «diaboliques ». Au Congo-Brazzaville, le musée de Kankata, où les objets recueillis par Malanda furent placés, a fait l'objet d'un pillage organisé. Ces objets ont traversé les mers, les océans et se sont retrouvés partout dans le monde pour enrichir aujourd'hui la vie intellectuelle des Européens et des Américains. Les prophètes Pascal et Malanda peuvent être considérés de ce point de vue comme des prophètes de malheur, car ils produisirent des objets de malheur qui sont de nos jours au cœur de la situation troublante où les intellectuels africains luttent pour le retour de ces mêmes objets.

9 Comme le suggèrent les observations qui viennent d'être faites, la double écriture entendue comme conjonction des écritures bibliques et comptables, était au fondement de la violence de l'imaginaire prophétique, et dans violence imaginaire, il y a le rôle important joué par les images. Toujours dans mes enquêtes congolaises, j'avais rencontré un prophète qui s'était proclamé "Télévision». Son nom traduisait une relation en miroir, si je puis dire, entre lui et les images télévisuelles : il était télévision car il disait voir très clair : il voyait ainsi tous les problèmes des gens qui venaient le consulter. Or, cet homme-télévision qui voit ses propres images dans une relation spéculaire avait été confronté à Monseigneur Pascal, au tout début de la carrière de ce dernier. En effet, d'après le récit que m'avait fait Monseigneur sur cette violente confrontation, tout commença par la décision prise par Monseigneur d'aller attaquer Jean Ndombakissa, devenu plus tard Télévision. La décision de cette attaque fut prise par Mademoiselle, dont le vrai nom était Élisabeth d'après Monseigneur lui-même. Le couple des esprits qui commandaient de l'intérieur Monseigneur était ainsi composé de Jean (le Baptiste) et d'Élisabeth. Dans ces années 1950, il n'était pas rare que sur les murs des maisons au Congo soient affichées les images tirées des magazines de mode ou de potins parisiens comme Ici Paris que les gens affectionnaient et parmi lesquelles les images des femmes blanches étaient particulièrement prisées. Le combat entre Jean Ndombakissa et le Monseigneur Pascal Zoaka Zoaka se déroula en grande partie par le biais de ces images. Pascal m'affirma avoir dit à Jean Ndombakissa: "Ces images qui sont sur tes murs vont se révolter et t'attaquer, elles vont te faire la guerre si tu ne jettes pas tous les fétiches que tu as, ces images vont te forcer à jeter tes fétiches ». Si, dans cette lutte entre des images, nous sommes au cœur de la visualité, il ne faut pas écarter que les images ont aussi une source auditive : le prophète entend des voix qu'il est capable de restituer, étant seul en mesure de dire ce que nous n'entendons pas. Mademoiselle (Élisabeth) parle en lui, Jean parle en lui, les images parlent en lui. C'est à partir de ces paroles des images, images mentales et images visuelles, que Monseigneur Pascal recréa le monde. En effet, les mouvements de défétichage qu'il entreprit sous le commandement de ces paroles que lui seul entendait produisirent des transformations dans la société : des objets de protection furent produits sous la violence de l'imaginaire 
de ces paroles comme des poisons psychiques. Écritures bibliques, écritures comptables, images des femmes blanches conspirèrent ainsi à la création de ce nouveau monde par l'entremise de la violence de l'imaginaire prophétique dont le fondement, comme nous l'avons vu plus haut, est la double écriture.

Lorsque je rencontrai Monseigneur Pascal dans les années 1990, il me dit: «Tu viens me voir mais tu dois dormir dans mon lit. On m'accuse d'être celui qui tue les gens et toi, je vais te tuer ». Je lui répondis que j'étais consentant, j'acceptais qu'il me tuât en passant la nuit dans son lit. Mon épouse fut effrayée par ma décision, mais elle ne put se résoudre à me laisser mourir seul dans le lit de Jean Ndombakissa qui, à l'époque, avait pris le nom de Professeur. Fatigués par la route, le sommeil eut raison des appréhensions de mon épouse et nous passâmes une nuit tranquille dans le lit du Professeur dont la réputation avait gagné les hautes sphères du pouvoir d'État au Congo et au Gabon. C'est ainsi qu'il fut souvent consulté par les grandes autorités du pouvoir marxiste de Brazzaville et par des politiques gabonais qui le firent voyager jusqu'à Libreville. Informée de mes relations avec le Professeur Jean Ndombakissa, une autorité de Brazzaville me sollicita pour l'accompagner jusqu'à celui-ci. Ce fut pour moi une occasion rêvée d'observer comment un marxiste-léniniste pouvait concilier l'idéologie du matérialisme historique avec le « fétichisme » qui en l'occurrence n'était pas celui de la marchandise.

11 Ces expériences de terrain me révélèrent combien la violence de l'imaginaire fondée sur la double écriture travaillait à la création de la réalité sous la pensée et la parole des images. Le Professeur Jean Ndombakissa n'eut pas seulement comme «collègue » le président Pascal Lissouba, qui lui aussi tenait absolument à son titre de Professeur dont il imposait la mention sur les documents officiels, mais aussi Mobutu Sese Seko, un autre créateur de réalité par la puissance des images télévisuelles. En effet, à Kinshasa, Joseph Désiré Mobutu, qui, par les vertus de l'authenticité zaïroise, avait changé son nom en Mobutu Sese Seko Nkunku Gbedu Waza Banga, s'autocréa Dieu à partir de la télévision. Dans le film de Thierry Michel, Mobutu roi du Zairre, on le voit sortir des nuages durant le journal télévisé, et cette image, selon Sakombi Inongo, le virtuose de la propagande de Mobutu, qui imagina cette mise en scène, avait si fortement frappé les esprits que des gens crurent réellement que Mobutu s'était divinisé. Ironie du sort, Sakombi Inongo qui après le décès de Mobutu mit ses talents au service de la propagande de Laurent-Désiré Kabila devint lui aussi un prophète-pasteur pentecôtiste.

Mais revenons à Mobutu pour montrer comment, par la double écriture, il travailla à la création d'une nouvelle réalité. En effet, en changeant son nom et en s'autocréant comme Dieu, il incarna l'éternité qui prenait immédiatement la signification de celle de son règne. C'est ainsi qu'il se dit: Les Blancs veulent une Constitution au Zaïre, alors donnons-leur ces écritures qui fixent les lois et imposent la Loi suprême. Comme dans le Royaume de Dieu où les Écritures sont la transcription de la parole de Dieu, il ne pouvait y avoir de différence entre la parole de Dieu-Mobutu et la Constitution. Il n'y a donc pas lieu de s'étonner que seul Dieu, l'autocréé, puisse s'autoriser de changer ses écritures. En fait, la Constitution est pour les autres et non pour lui, le SouverainMobutu, qui la dicte et l'inspire. Point n'est besoin de s'y conformer, cette Constitution circonstancielle n'étant destinée qu'à calmer ceux qui ont foi dans l'écriture ; lui n'a foi qu'en lui-même puisqu'il est Dieu?

13 De plus, en tant que Dieu créateur, il n'est pas soumis à l'interdit de l'inceste : ses femmes sont forcément ses créatures, comme le sont le Zaïre, le fleuve Zaïre, la 
monnaie Zaïre et les citoyens zaïrois parmi lesquels ses propres parents. Ainsi, en sa qualité de créateur de tout et de tous, il est le père de son père et le père de sa mère, et en même temps le mari de sa mère, etc. Les deux jumelles qu'il va épouser à la suite de la mort de sa première épouse ${ }^{8}$ correspondent à la dimension divine de son pouvoir car les jumelles sont investies, comme toutes les figures du double, par un imaginaire de la puissance mystique. Elles sont de fait à la fois épouses et filles de Mobutu. Il s'ensuit que les mâles du pays, qui n'en restent pas moins ses créatures, deviennent ses rivaux car ils se disputent les mêmes femmes.

Qu'en est-il de l'anthropologue, obligé de suivre ce que crée le prophète ? Nul doute que celui-ci est en dette permanente, d'une dette inexhaustible puisque ce n'est pas lui qui crée la dette. N'étant que le lecteur de la réalité créée par l'autre, cet " autre qui crée " et auprès duquel il sera toujours débiteur, sa situation de dette n'a de cesse de s'étendre à tous ces gens qui créent la réalité et envers lesquels il sera invariablement redevable, bien que certains, comme Jean-Pierre Dozon, arguent que le prophète est le collègue de l'anthropologue.

15 J'ai avancé au début de cette contribution que subsistait un reste inépuisable du discours de l'Autre, créateur de réalité que le sociologue ou l'anthropologue ne pouvaient dompter. C'est la conscience de cette limite, qui s'impose consciemment ou peut-être surtout inconsciemment dans l'esprit du chercheur scientifique, qui le pousse à se lancer lui-même dans cette aventure de la double écriture : écriture du scientifique qui se double de l'écriture du romancier. C'est ce qui m'est arrivé personnellement en publiant deux œuvres de fiction : Chiens de foudre ${ }^{9}$ et Tuée-tuée mon amour ${ }^{10}$. De l'avis des critiques littéraires, ces deux textes sont la traduction de la relation entre les imaginaires et la connaissance scientifique ${ }^{11}$. Mais le syndrome de la double écriture qui affecte aussi bien les prophètes, les chefs d'État et les chercheurs, qui est aussi la trace de l'inextricable rapport entre le réel et l'imaginaire, la réalité et la fiction, s'est manifesté de cette manière : la création par un artiste d'une fiction fécondée par mon travail de sociologue et d'anthropologue. En effet, après une conférence que j'avais présentée à Dakar en mai 2018, sur les thèmes des éblouissements, l'artiste sud-africain François Koetze, qui était dans le public et qui avait tout enregistré, a produit une œuvre de fiction, Core Dump ${ }^{12}$. C'est ainsi que la création de la réalité par le prophète, sous le commandement des esprits en lui, mise en forme manuscrite par le sociologue, sous le commandement de l'esprit scientifique en lui, lequel souffrait de l'excès de la création prophétique au point de penser en moi et de m'amener à me lancer dans l'écriture romanesque, dut parler en l'artiste François Koetze. Celui-ci, inspiré par ma parole, créa Core Dump, redoublant ainsi la force de mes manuscrits scientifiques et littéraires, eux-mêmes inspirés par la réalité créée, comme je viens de le dire, par l'imaginaire prophétique.

\section{NOTES}

1. Joseph Tonda, «Le syndrome du prophète », Cahiers d'études africaines, vol. 41, n 161, 2001, p. 139-162. 
2. Pierre Bourdieu, Sociologie générale, vol. 1, Cours au Collège de France, 1981-1983, Raisons d'agir, Paris, 2015, p. 295.

3. Joseph Tonda, Le Souverain moderne. Le corps du pouvoir en Afrique centrale (Congo, Gabon), Paris, Karthala, 2005.

4. Joseph Tonda, La Guérison divine en Afrique centrale (Congo, Gabon), Paris, Karthala, 2002.

5. Si Izanga est le terme générique pour dire sorcellerie, Ngoyi est l'autre terme qui signifie précisément panthère. Izanga la Ngoyi signifie la « sorcellerie de la panthère », celle des personnes censées se transformer en panthère la nuit, notamment dans leur « sommeil ».

6. J'en ai parlé dans la conclusion de La Guérison divine en Afrique centrale, op. cit.

7. Patrice Yengo, «L'ordre de la transgression. Imaginaire du pouvoir, souveraineté postcoloniale et "démocratie" en Afrique centrale ", Sociétés politiques comparées, 45, mai-août 2018.

8. Une mort qui lui est, au demeurant, attribuée.

9. Joseph Tonda, Chiens de foudre, Libreville, ODEM, 2013.

10. Joseph Tonda, Tuée tuée mon amour, Libreville, La Doxa Éditions, « La librevilloise », 2017.

11. Cf. Noël Bertrand Boundzanga, Christ-Olivier Mpaga, Placide Ondo (éd.), Joseph Tonda. Entre imaginaires et connaissance, Paris, L'Harmattan, 2019.

12. Core Dump est une série de 4 films, Kinshasa (2018), Shenzhen (2019), New York (2019) et Dakar (2018) dont le projet explore la relation entre la technologie numérique, la cybernétique, le colonialisme et la notion réenchantée d'une utopie humaniste. https://francoisknoetze.com/ core-dump-2018/https://francoisknoetze.com/core-dump-2018/

\section{RÉSUMÉS}

Les prophètes sont des producteurs de récits que les sciences humaines se préoccupent de convertir en récits scientifiques, exprimant ainsi leur inextinguible dette à l'endroit de ces créateurs de réalités. Et c'est parce que cette dette est inextinguible, que le sociologue et l'anthropologue, hantés par le syndrome du prophète, éprouvent au fond d'eux-mêmes une douloureuse insatisfaction de leur travail de conversion de la réalité créée par le geste prophétique en objet de connaissance scientifique. Il y a en effet ce reste inépuisable du discours de l'Autre créateur de réalité que l'on ne saurait dompter qu'en se lançant soi-même dans cette aventure de la double création, qui peut prendre la forme de la fiction littéraire, ou celle du film documentaire ethnographique que l'on produit soi-même ou que la production du scientifique inspire, redoublant ainsi la force de ses manuscrits. Entreprise très risquée, disent les spécialistes. Et pourtant, entreprise d'une longue histoire, que Jean Rouch, par exemple, a marqué de son empreinte. Peut-être que finalement, l'ethnologue, le sociologue, l'anthropologue, l'écrivain et le prophète sont-ils tous, par leur travail en double, des Maîtres fous?

\section{INDEX}

Mots-clés : prophète, anthropologue, ça, violence, afrodystopie, images, écritures, fétiche, dette 
AUTEUR

JOSEPH TONDA

Professeur de sociologie, d'anthropologie et romancier, Université de Libreville, Gabon. 